
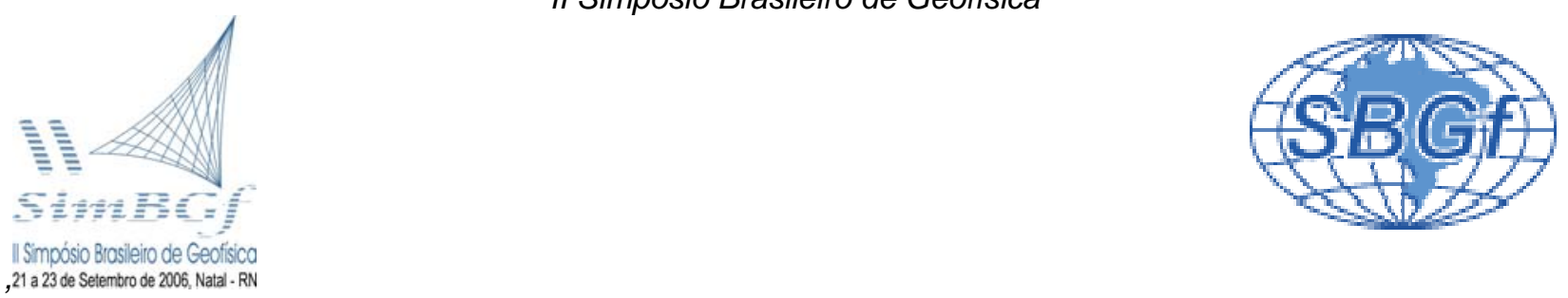

\title{
Migração Reversa no Tempo Aplicada a Dados Sísmicos Levantados no Talude Continental da Região de Camamu (BA)
}

Antônio Vladimir Moura Lima, Marco Antônio B. Botelho, CPGG/UFBA.

Copyright 2006, SBGf - Sociedade Brasileira de Geofísica

Este texto foi preparado para a apresentação no II Simpósio de Geofísica da Sociedade Brasileira de Geofísica, Natal/RN, 21-23 de setembro de 2006. Seu conteúdo foi revisado pela Comissão Tecno-científica do II SR-SBGf mas não necessariamente representa a opinião da SBGf ou de seus associados. É proibida a reprodução total ou parcial deste material para propósitos comerciais sem prévia autorização da SBGf.

\section{Resumo}

Neste trabalho construímos um algoritmo para fazer a extrapolação direta no tempo (modelagem direta) e a retropropagação no tempo para realizar migração reversa no tempo, tanto para dados sísmicos sintéticos ou reais. Com o objetivo de obter um modelo representativo da geometria das estruturas geológicas presentes na zona de quebra de talude continental na região de Camamu (Bahia), usamos a migração reversa no tempo (Reverse Time Migration, RTM) para criar o modelo em profundidade, a partir de uma seção de afastamento constante (afastamento de $300 \mathrm{~m}$ ) a qual é tratada como se fosse afastamento nulo. $O$ algoritmo de migração utiliza a equação acústica da onda, cujas derivadas são resolvidas por intermédio do método das diferenças finitas, fazendo-se uso dos operadores de segunda e quarta ordem para aproximar as segundas derivadas no tempo e no espaço, respectivamente.

\section{Introdução}

A sísmica de reflexão usada na exploração de hidrocarbonetos tem como finalidade a obtenção de modelos de subsuperfície, seja para determinar estruturas que funcionam como reservatórios de hidrocarbonetos ou simplesmente obter uma imagem confiável da subsuperfície para estudos acadêmicos.

Atualmente existem diversas técnicas de modelagem direta, e diferentes tipos de migrações antes e após o empilhamento que podem ser usado para testar modelos oriundos do processamento e interpretação de dados sísmicos. Os métodos de migração fornecem uma aproximação do modelo geométrico de subsuperfície. Neste trabalho realizou-se modelagem direta de um modelo simples com variação lateral de velocidade, bem como a migração RTM de dados sísmicos sintéticos e reais.

A migração reversa no tempo de dados sísmicos empilhados tem sido descrita na literatura há mais de duas décadas. Os trabalhos de Baysal et. al (1983), McMechan (1983), Loewenthal e Mufti (1983), Whitmore (1983) e Levin (1984) foram pioneiros na utilização de RTM para migrar dados sísmicos empilhados.
A aplicação da RTM em dados com afastamento fontereceptor variável, surgiu posteriormente com os trabalhos de Reshef e Kosloff (1986) e Chang e McMechan (1986), sendo que este último empregou operadores de diferenças finitas para solucionar a equação acústica da onda, tal como foi feito neste trabalho.

\section{Metodologia/ Problema Investigado}

As diversas técnicas de migração diferem apenas entre quanto ao modo como são implementadas e aos métodos numéricos utilizados, porém sempre envolvem duas etapas: a extrapolação e a construção da imagem. Existem diferentes métodos de extrapolação, alguns simples outros mais sofisticados. Todos, entretanto, são derivados de alguma forma da equação acústica ou elástica da onda e dependem mais ou menos de uma aproximação de alta freqüência (Han, 2000). Como na aquisição convencional, ainda amplamente utilizada pela extrapolação sísmica, registram-se preferencialmente as ondas compressionais, a maioria dos métodos de migração empregam a equação acústica da onda.

O método RTM (Reverse Time Migration) difere dos métodos espectrais por ser mais robusto aceitando qualquer variação lateral de velocidade e realizando a migração por meio de uma depropagação no tempo do campo de ondas. Ele realiza uma depropagação no tempo, do campo de ondas, ou melhor, calcula a posição espacial dos pontos de reflexão do campo de ondas em profundidade, usando o tempo de imagem, o qual no caso da seção empilhada corresponde a $t=0$, segundo o modelo do refletor explosivo. Ao final do processo de migração a seção em tempo $S(x, z=0, t)$ fornecerá a seção em profundidade $S(x, z, t=0)$, correspondente ao modelo geométrico/geológico. Caso a seção tenha o afastamento fonte-receptor variável (família de tiro comum ou família CMP), a migração é feita com o auxílio de uma matriz tempo de imagem que corresponde ao tempo gasto pela frente de onda descendente a partir da fonte até cada nó da malha. Esta metodologia permite recuperar a verdadeira posição de refletores sem restrições a qualquer geometria.

A equação acústica da onda é uma equação diferencial parcial linear de segunda ordem que possui duas soluções independentes, ou dois campos contínuos no espaço e no tempo, que são os campos de pressão e o da velocidade da partícula. O campo de pressão é representado por um campo escalar, enquanto o da velocidade da partícula por um campo vetorial. A equação acústica da onda é obtida utilizando-se de duas 
relações. A primeira é a lei de Hooke, formulada da teoria da elasticidade, na qual é estabelecido que as deformações que ocorrem em um corpo são proporcionais às tensões que provocam tais deformações, ou seja:

$$
U=-k(\nabla \cdot u)
$$

A segunda é a lei de Newton também conhecida como equação do movimento, e estabelece que a força é proporcional à aceleração:

$$
\rho \frac{\partial^{2} u}{\partial t^{2}}=-\nabla \cdot U
$$

Utilizando de alguns artifícios matemáticos podemos chegar a equação acústica da onda:

$$
\nabla^{2} U=\frac{1}{v^{2}} \frac{\partial^{2} U}{\partial t^{2}}
$$

que pode ser apresentada da seguinte forma:

$$
\frac{\partial^{2} U}{\partial x^{2}}+\frac{\partial^{2} U}{\partial y^{2}}+\frac{\partial^{2} U}{\partial z^{2}}=\frac{1}{v^{2}} \frac{\partial^{2} U}{\partial t^{2}}
$$

Utilizando operadores diferenciais de quarta e segunda ordem para as derivadas espaciais e temporais respectivamente (eq.1.5, eq.1.6, eq.1.7 e eq.1.8), podemos discretizar o espaço $(x, y, z)$ em cubos (caso 3D) fig.1.1 ou em retângulos (caso 2D), possibilitando desta forma obter a equação da onda na forma discreta, vide eq. 1.9:

$$
\begin{gathered}
U_{x x}=\frac{1}{12(\Delta x)^{2}}\left(-U_{k-2, m, n}^{t}+1 U_{k-1, m, n}^{t}-3 \bigcup_{k, m, n}^{t}+1 U_{k+1, m, n}^{t}-U_{k+2, m, n}^{t}\right), \\
U_{y y}=\frac{1}{12(\Delta y)^{2}}\left(-U_{k, m-2, n}^{t}+1 U_{k, m-1, n}^{t}-3 \bigcup_{k, m, n}^{t}+1 U_{k, m+1, n}^{t}-U_{k, m+2, n}^{t}\right), \\
U_{z z}=\frac{1}{12(\Delta v)^{2}}\left(-U_{k, m, n-2}^{t}+1 U_{k, m, n-1}^{t}-3 U_{k, m, n}^{t}+1 U_{k, m, n+1}^{t}-U_{k, m, n+2}^{t}\right), \\
U_{t t}=\frac{1}{12(\Delta t)^{2}}\left(U_{k, m, n}^{t-1}-2 U_{k, m, n}^{t}+U_{k, m, n}^{t+1}\right),
\end{gathered}
$$$$
U_{k, m, n}^{t+1}=2 U_{k, m, n}^{t}-U_{k, m, n}^{t-1}-\left[A_{x}+A_{y}+A_{z}\right] 2,5 U_{k, m, n}^{t}+
$$$$
+\frac{A_{x}}{12}\left[-U_{k-2, m, n}^{t}+16 U_{k-1, m, n}^{t}+16 U_{k+1, m, n}^{t}-U_{k+2, m, n}^{t}\right]+
$$$$
+\frac{A_{y}}{12}\left[-U_{k, m-2, n}^{t}+16 U_{k, m-1, n}^{t}+16 U_{k, m+1, n}^{t}-U_{k, m+2, n}^{t}\right]+
$$$$
\frac{A_{z}}{12}\left[-U_{k, m, n-2}^{t}+16 U_{k, m, n-1}^{t}+16 U_{k, m, n+1}^{t}-U_{k, m, n+2}^{t}\right]
$$

Desta forma torna-se possível proceder a modelagem direta (propagação), bem como migrar (retro-propagação) dados com diferentes configurações, desde quando respeite-se os limites de estabilidade do método préestabelecido, já que a utilização de operadores de diferenças finitas para a solução da equação da onda é um processo estável dentro de determinados limites. Faria (1986) demonstrou matematicamente o procedimento para obtenção dos limites de estabilidade do processo de diferenças finitas para a solução da equação acústica chegando a:

$$
A_{x}+A_{y}+A_{z} \leq \frac{3}{4}
$$

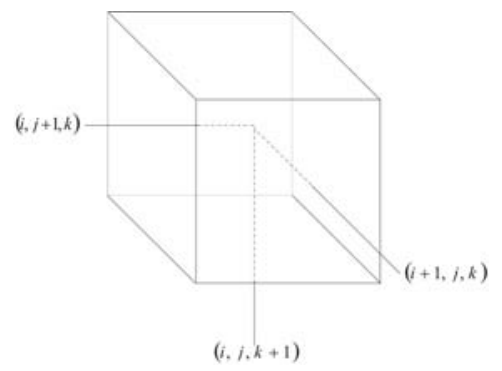

Figura 1.1 - Cubo discretizado no espaço $(x, y, z)$

\section{Resultados}

Inicialmente aplicou-se a migração em dados sintéticos, todos apresentando um refletor horizontal subjacente, de forma a testar o algoritmo de migração quanto a sua capacidade de reconstruir a verdadeira posição espacial dos refletores subjacentes. O algoritmo foi testado com sucesso em uma seção de afastamento nulo gerada sob o conceito do "refletor explosivo" empregando os operadores de diferenças finitas e a equação acústica da onda para simular a propagação do pulso; e também na seção de afastamento nulo gerada a partir do modelo de sal disponibilizado pela EAGE (European Association of Geoscientists and Engineers). A seção sintética (vide figura 1.3) tipo "refletor explosivo", a qual será posteriormente migrada, foi gerada a partir do campo de velocidades da figura 1.2 criado com auxilio do SUSeismic Unix (v1=2200 m/s, 3050m/s, $2200 \mathrm{~m} / \mathrm{s})$. O resultado da migração RTM é mostrado na figura 1.4. No segundo modelo do domo salino, proposto pela SEGEAGE, cujo campo de velocidades esta apresentado na figura 1.5, a seção de afastamento nulo na figura 1.6 e a seção migrada final recuperando todas as feições geométricas abaixo do corpo de sal, vide figura 1.7. A terceira aplicação da RTM foi numa seção de afastamento mínimo $(x=300 \mathrm{~m})$ real, registrada sobre o talude continental da Região de Camamu (BA). Como não sabemos o correto campo de velocidades para migrar o dado real, realizamos uma primeira migração com a velocidade da onda, na água ( $\mathrm{V}=1500 \mathrm{~m} / \mathrm{s})$. Definindo o correto posicionamento do assoalho oceânico. Uma segunda migração RTM empregou a velocidade do meio $1500 \mathrm{~m} / \mathrm{s}$ para velocidade acima do refletor correspondente ao assoalho oceânico e $3000 \mathrm{~m} / \mathrm{s}$ para a porção abaixo. A seção real de afastamento 
comum pode ser considerada, para qualquer efeito, uma seção empilhada (aproximação) principalmente nas porções mais profundas de lâmina d'água $(z=1,5 \mathrm{Km})$. Após a segunda migração foi possível obter uma boa imagem (vide Figura 1.9) ressaltando eventos logo abaixo do refletor correspondente ao assoalho e demonstrando que a metodologia empregada mostra-se bastante eficaz. O resultado da migração pode ser refinado usando procedimentos de migração em cascata como apresentado em Botelho (1986) de forma a obter cada vez mais uma imagem precisa da região estudada. Segue alguns resultados obtidos com tal técnica:

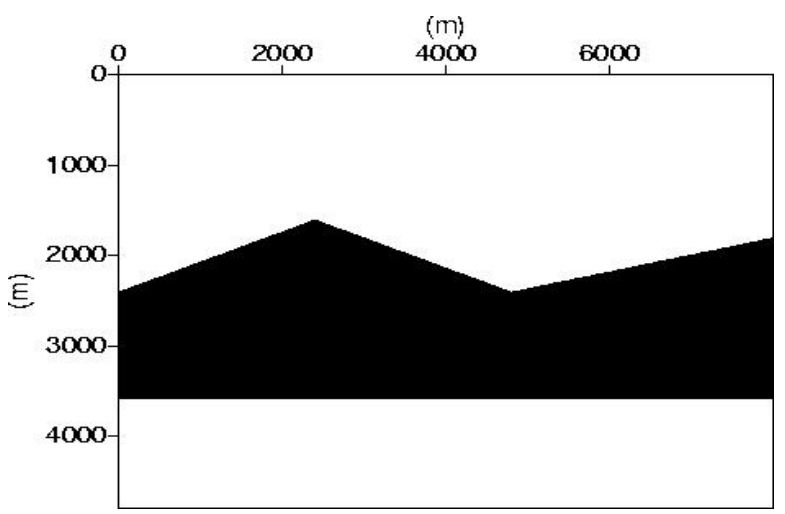

Figura 1.2 - Campo de velocidade sintético (Velocidade de cima para baixo: 2200 m/s, 3050 m/s, 2200 m/s).

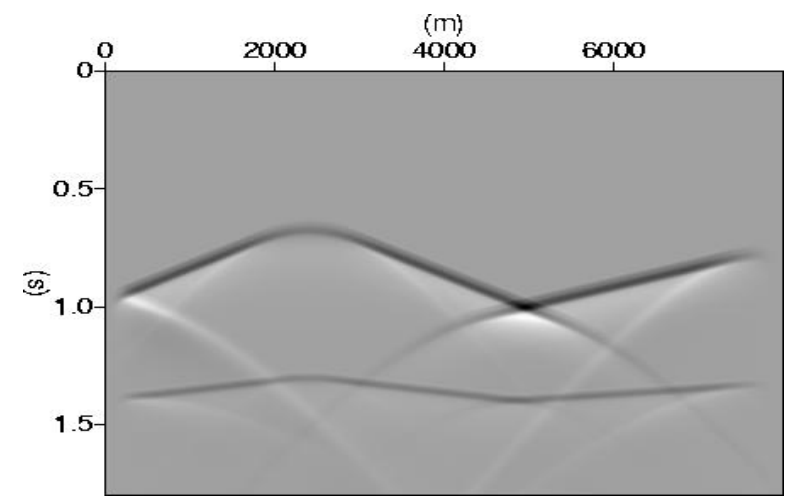

Figura 1.3 - Seção em tempo referente ao modelo da figura1. 2.

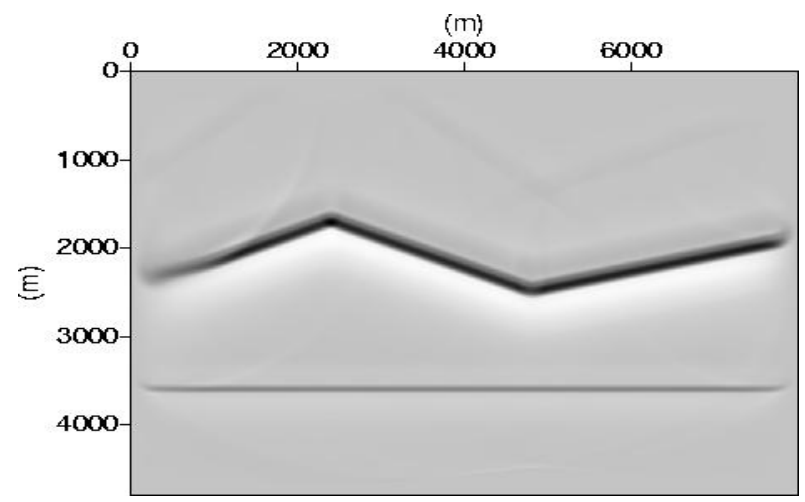

Figura 1.4 - Seção migrada referente a seção em tempo da figura 1.3 e campo de velocidade da figura 1.2.

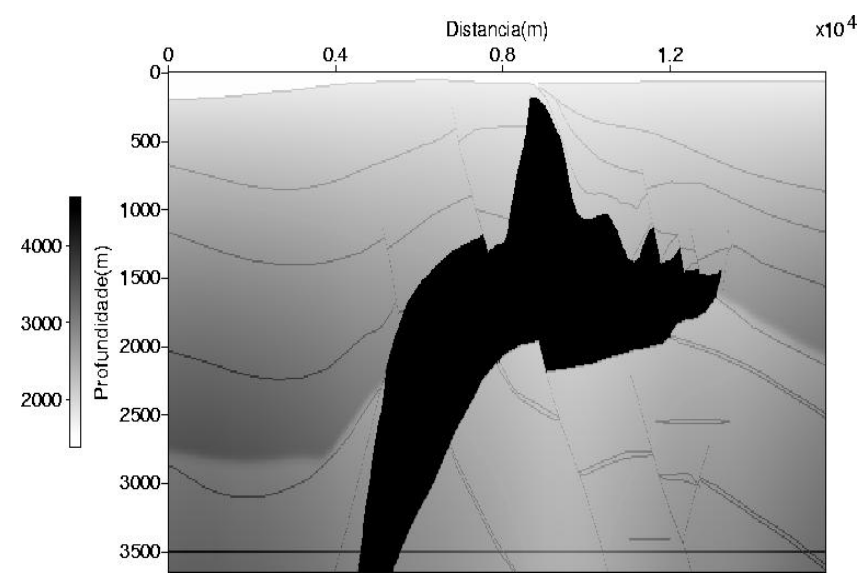

Figura 1.5 - Campo de velocidade referente ao modelo do domo salino SEG-EAGE.

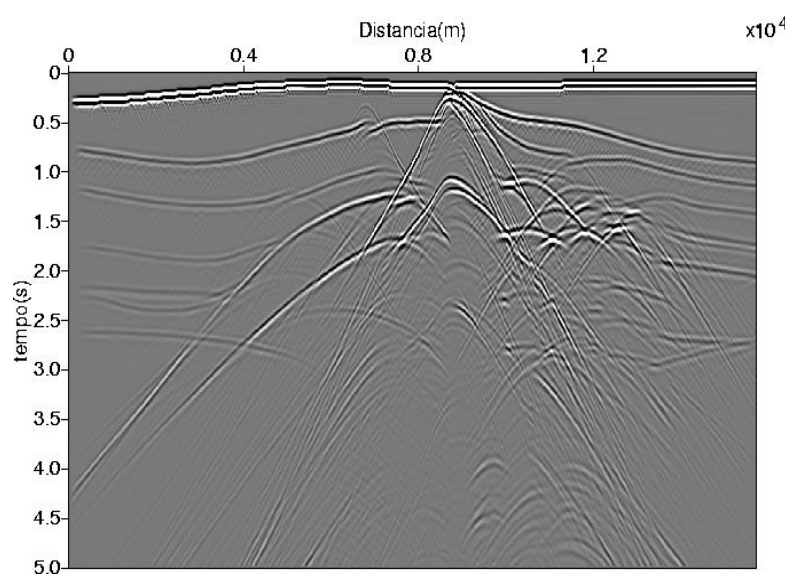

Figura 1.6 - Seção em tempo referente ao modelo do domo salino.

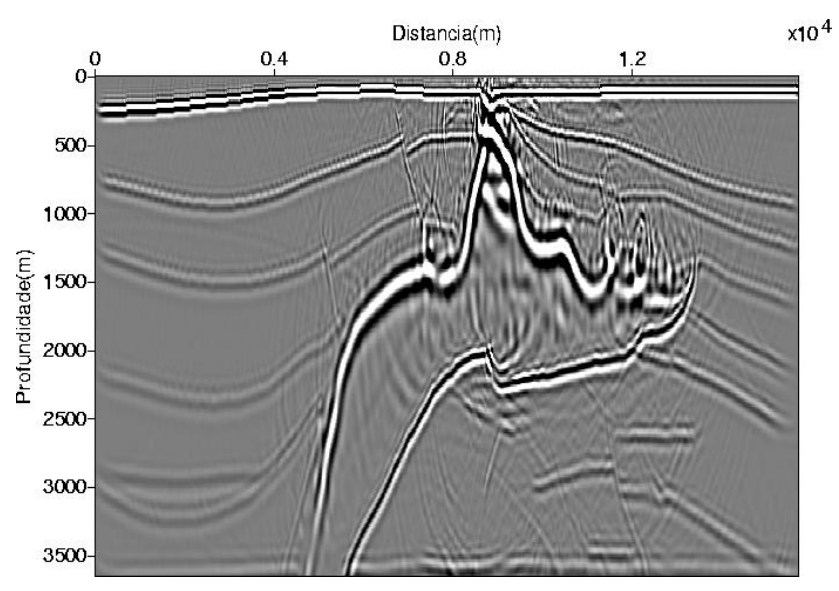

Figura 1.7 - Seção migrada utilizando migração reversa no tempo e malha retangular no espaco. 


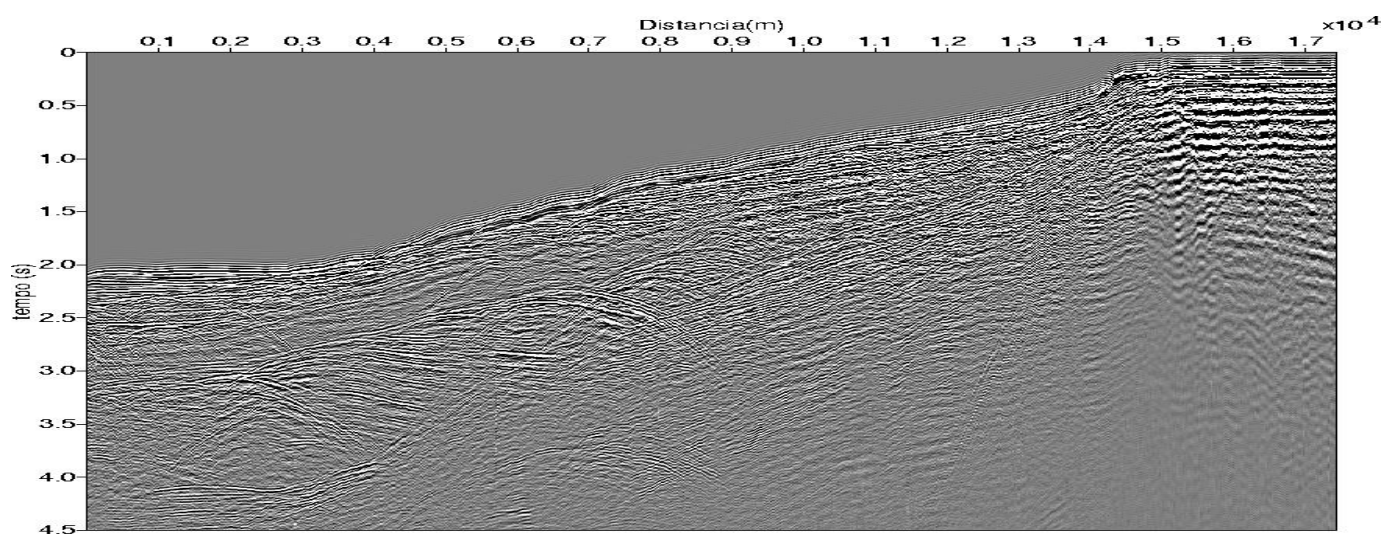

Figura 1.8 - Seção de afastamento comum do Talude Continental da Região de Camamu (BA) igual a $300 \mathrm{~m}$.

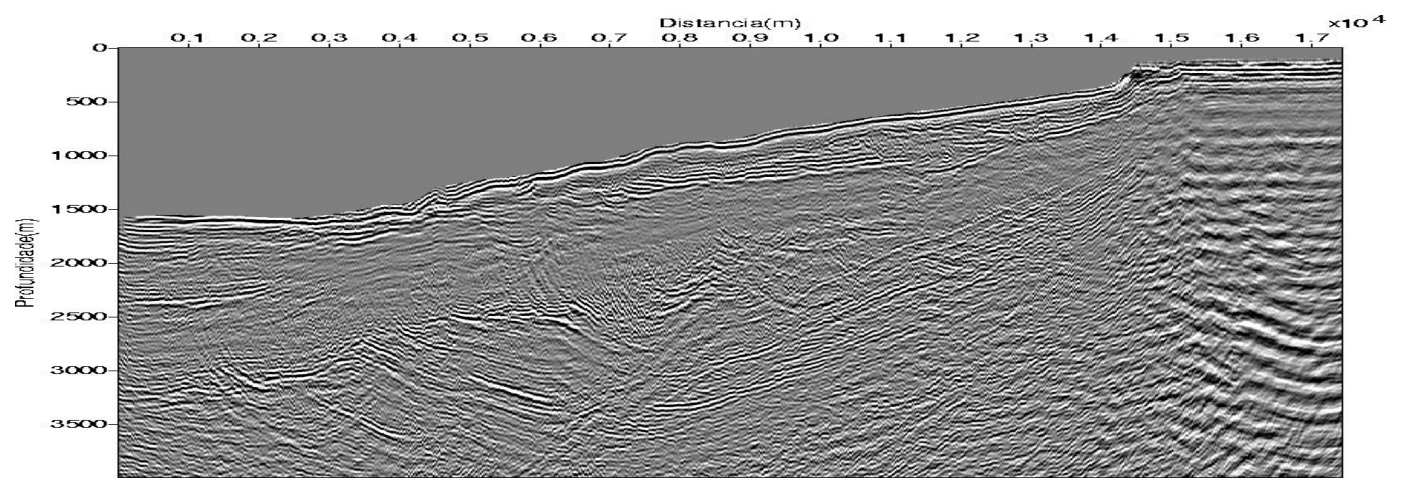

Figura 1.9 - Seção migrada referente ao dado real do talude Continental da Região de Camamu (BA)

\section{Discussão e Conclusões}

A técnica de migração reversa no tempo consegue recuperar de forma fidedigna diversas estruturas geológicas sem restrições quanto a qualquer inclinação dos refletores presentes. Os recursos computacionais hoje disponíveis diminuem o tempo de processamento requerido, tornando o método mais robusto e competitivo em relação aos métodos espectrais comumente utilizados na área. O modelo sintético do domo de sal, fornecido pelo consórcio EAGE-SEG, teve um refletor horizontal, abaixo do domo irregular de sal reconstituído pela migração RTM da seção de afastamento nulo correspondente ao citado modelo. A migração RTM comprova que pode lidar com contrastes bruscos de velocidade em qualquer direção. A migração da seção de afastamento constante $(x=300 \mathrm{~m})$ forneceu uma imagem onde poderá vislumbrar depósitos sub-horizontais mais recentes e até blocos falhados deslocados subjacentes.

\section{Agradecimentos}

Os autores agradecem ao PRH-08/ANP pelo financiamento na forma de Bolsa de Estudo concedida ao primeiro autor e convenio CTPETRO/CNPQ-FINEP Fase 1 e Fase 2 pelo suporte dado a esta pesquisa.

\section{Referências}

BAYSAL, E., KOSLOFF, D., AND SHERWOOD, J. W. C., 1983, Reverse Time Migration, Geophysics, 48, 15141524

BOTELHO, M. A. B., 1986, Modelamento Sísmico na Bacia do Recôncavo Usando a Técnica de Traçamento dos Raios, CPGG/UFBA - Tese de Doutorado.

BOTELHO. B.., and Stoffa, p.L., 1991, Finite-Difference Reverse Time Migration of Multi-Configuration marine seismic data, $2^{\circ}$ Congresso Internacional da SBGF, Vol.22, 953-955

FARIA, E L 1986, Migração Antes do Empilhamento Utilizando Propagação Reversa no Tempo, CPGG/UFBA - Dissertação de Mestrado.

LEVIN A., 1984, Principles of Reverse Time Migration, Geophysics, 48, 627-635

LOEWENTHAL, D., and Mufti, I. R., 1983, Reverse time Migration in the spatial frequency domain, Geophysics, 48, 627-635

MCMECHAN, G. A., 1983, Migration by extrapolation of time dependent boundary values, Geophysics Prospecting, 31, 413-420

RESHEF AND KOSLOFF. Migration of common shot gathers. Geophysics, 51:324--331, 1986. 